\title{
Practical Stability and Integral Stability for Singular Differential Systems with Maxima
}

\author{
Junyan Bao $\mathbb{D}$, Peiguang Wang $(\mathbb{D}$, and Yanjun Li \\ College of Mathematics and Information Science, Hebei University, Baoding 071002, China \\ Correspondence should be addressed to Peiguang Wang; pgwang@hbu.edu.cn
}

Received 17 February 2020; Accepted 14 March 2020; Published 14 April 2020

Guest Editor: Rongwei Guo

Copyright (C) 2020 Junyan Bao et al. This is an open access article distributed under the Creative Commons Attribution License, which permits unrestricted use, distribution, and reproduction in any medium, provided the original work is properly cited.

In this paper, we introduce various definitions of practical stability and integral stability for nonlinear singular differential systems with maxima and give criteria of stability for such systems via the Lyapunov method and comparison principle.

\section{Introduction and Preliminaries}

Differential equations with maxima are a special type of differential equations that contain the maximum of the unknown function over a previous interval, of which many examples are found in the fields of application such as automatic control, population dynamics, disease control, and so on. Recently, the research interest in differential equations with maxima has increased exponentially. Some stability results for such equations can be found in the monographs $[1,2]$, the papers [3-9], and references cited therein.

In practical applications, many problems can be described by singular system models, such as optimal control problems and constrained control problems, which can be found in the monographs of Campbell [10] and Dai [11]. Singular system is a type of dynamic system which is more complicated than the ordinary one. Owing to its complicated structure and many other factors, the study of stability for singular systems involves greater difficulty than that of nonsingular systems. Till now, various types of stability for singular systems have been investigated via Lyapunov functions. However, most previous studies focused on the singular systems described by ordinary differential equations [10-13], difference equations [14-17], and delay differential equations [18-20], and there are a few results for singular differential systems with maxima. In addition, differential equations with maxima have some different properties from the well-known differential equations and delay differential equations.

The purpose of this paper is to integrate these two areas and analyze the practical stability and integral stability of nonlinear singular systems with maxima. To extend Lyapunov's stability and support the specific needs of singular systems, we introduce the function $q(t, x)$ and obtain some different types of stability criteria by using the Lyapunov function method and comparison principle.

\section{Practical Stability}

The practical stability, being quite different from the stability in the sense of Lyapunov, is neither weaker nor stronger than the usual stability. It is significant from the perspective of engineering application (see [21-25]). In this section, by using Lyapunov functions and the comparison principle, we study some practical stability for the following singular differential systems.

Consider the singular differential systems with maxima

$$
\begin{cases}E \dot{x}=f\left(t, x, \max _{s \in[t-\tau, t]} x(s)\right), & \text { for } t \geq t_{0} \geq 0, \\ x_{t_{0}}=\varphi(t), & t \in[-\tau, 0],\end{cases}
$$

where $E \in R^{n \times n}$ with $\operatorname{rank}(E)<n$ is a singular constant matrix, $x \in R^{n}, f \in C\left(R_{+} \times R^{n} \times R^{n}, R^{n}\right), f(t, 0,0) \equiv 0, \tau>0$ is a constant, and $\varphi \in C\left([-\tau, 0], R^{n}\right)$. 
Firstly, we introduce the following notations and sets for convenience.

Let $T_{k}=\left[0, t_{k}\right)$, where $0<t_{k} \leq+\infty ; q(t, x) \in C^{1}(J \times$ $\left.R^{n}, R^{m}\right), q(t, 0) \equiv 0, J \subseteq R_{+} . S_{k}\left(t_{0}\right)$ is a set of all consistent initial functions at initial time $t_{0}$. Then, for any $\varphi \in S_{k}\left(t_{0}\right)$, there exists at least one continuous solution of systems (1) in $\left[t_{0}-\tau,+\infty\right)$ through $\left(t_{0}, \varphi\right)$ (see [20]).

$$
\begin{aligned}
& K=\left\{a(t) \in C\left(T_{k}, R_{+}\right) \mid a(t) ;\right. \\
& \text { is strictly increasing and } a(0)=0\} \\
& K^{*}=\left\{a\left(t_{0}, r\right) \in C\left(T_{k} \times R_{+}, R_{+}\right) \mid a\left(t_{0}, r\right) ;\right. \\
& \text { is strictly increasing in } \left.r \text { and } a\left(t_{0}, 0\right)=0\right\}
\end{aligned}
$$

$$
Q(\varepsilon)=\left\{x \in R^{n} \mid\|q(t, x)\|<\varepsilon, t \in T_{k}, \varepsilon>0 \text { is a constant }\right\}
$$

$$
\begin{aligned}
& D(A)=\left\{x \in R^{n} \mid\|q(t, x)\|<A, A>0 \text { is a constant }\right\} \\
& \Lambda=\left\{V \mid V \in C\left(R \times D(A), R_{+}\right)\right. \text {and } \\
& V \text { islocallyLipschitzianin } x\} \\
& G \Longrightarrow H \text { means that } G \text { implies } H
\end{aligned}
$$

We denote by $x(t) \equiv x\left(t ; t_{0}, \varphi\right)$ the solution of the initial value problems (1).

Definition 1. Let $V \in \Lambda, t \in T_{k}$, and we define the derivative of the function $V(t ; x)$ along the trajectory of solution of the singular systems (1) as follows:

$$
D_{(2.1)}^{+} V(t, x(t))=\limsup _{h \longrightarrow 0} \frac{1}{h}\left\{V\left(t+h, x(t)+h f\left(t, x(t), \max _{s \in[-\tau, 0]} x(t+s)\right)\right)-V(t, x(t))\right\} .
$$

Definition 2. Let $\varphi \in S_{k}\left(t_{0}\right)$. The singular systems (1) is said to be

$\left(\mathrm{S}_{1}\right)$ stable with respect to $\left(q(t, x), T_{k}\right)$ if for any $\varepsilon>0$, and some $t_{0} \in T_{k}$, there exists $\delta\left(t_{0}, \varepsilon\right)>0$, such that

$$
\begin{array}{r}
\max _{s \in[-\tau, 0]}\|\varphi(s)\|<\delta\left(t_{0}, \varepsilon\right) \Longrightarrow\left\|q\left(t, x\left(t ; t_{0}, \varphi\right)\right)\right\|<\varepsilon \\
\text { for } t \geq t_{0} .
\end{array}
$$

Definition 3. Let $\varphi \in S_{k}\left(t_{0}\right)$. The singular systems (1) is said to be $\left(P S_{1}\right)$ practically stable for given $(\lambda, A)$ with $0<\lambda<A$ and some $t_{0} \in T_{k}$, such that

$$
\begin{array}{r}
\max _{s \in[-\tau, 0]}\|\varphi(s)\|<\lambda \Longrightarrow\left\|q\left(t, x\left(t ; t_{0}, \varphi\right)\right)\right\|<A \\
\text { for } t \geq t_{0}
\end{array}
$$

$\left(P S_{2}\right)$ uniformly practically stable if $\left(P S_{1}\right)$ holds for all $t_{0} \in T_{k}$

$\left(P_{3}\right)$ practically quasistable for given $(\lambda, B, T)$ with $\lambda, B, T>0$, and some $t_{0} \in T_{k}$, we have

$$
\begin{array}{r}
\max _{s \in[-\tau, 0]}\|\varphi(s)\|<\lambda \Longrightarrow\left\|q\left(t, x\left(t ; t_{0}, \varphi\right)\right)\right\|<B, \\
\text { for } t \geq t_{0}+T,
\end{array}
$$

$\left(P S_{4}\right)$ uniformly practically quasistable if $\left(P S_{1}\right)$ holds for all $t_{0} \in T_{k}$

$\left(P S_{5}\right)$ strongly practically stable if $\left(P S_{1}\right)$ and $\left(P S_{3}\right)$ hold simultaneously

$\left(P S_{6}\right)$ strongly uniformly practically stable if $\left(P S_{2}\right)$ and $\left(\mathrm{PS}_{4}\right)$ hold simultaneously

Remark 1. If $q(t, x)=x, t_{k}=+\infty$, and $S_{k}\left(t_{0}, t_{k}\right)=C([-\tau$, $\left.0], R^{n}\right)$, then Definitions 2 and 3 reduce to the concepts of classic Lyapunov stability.
It is well known that the comparison principle plays an important role in the development of stability theory. By the comparison principle, we can reduce the study of a given complicated differential system to that of a relatively simpler differential equation. For this purpose, we give the following lemma and definition.

Lemma 1 (See [1]). Assume that the following conditions hold

$\left(A_{1}\right) m(t) \in C\left(R_{+}, R_{+}\right), g(t, u) \in C\left(R_{+} \times R_{+}, R\right)$ and for any $t \in T_{k}$ such that $m(t)>m(t+s)$ for $s \in[-\tau, 0)$, the inequality

$$
D^{+} m(t) \leq g(t, m(t))
$$

holds, where $D^{+} m(t)=\lim \sup _{h \longrightarrow 0^{+}}(1 / h)[m(t+h)-$ $m(t)], g(t, 0) \equiv 0$

$\left(A_{2}\right)$ the maximal solution $r(t) \equiv r\left(t ; t_{0}, u_{0}\right)$ of the scalar equation

$$
\dot{u}=g(t, u), u\left(t_{0}\right)=u_{0},
$$

exists, on $\left[t_{0},+\infty\right)$. Then, $m(t) \leq r(t), t \geq t_{0}$, provided $\max _{s \in[-\tau, 0]} m\left(t_{0}+s\right) \leq u_{0}$.

Definition 4. Comparison equation (7) is said to be $\left(P S_{7}\right)$ practically stable if for given $(\lambda, A)$ with $0<\lambda<A$ and some $t_{0} \in R_{+}$, we have $u_{0}<\lambda$ implies $u(t)<A$, for $t \geq t_{0}$

$\left(P S_{8}\right)$ uniformly practically stable if $\left(P S_{7}\right)$ holds for all $t_{0} \in R_{+}$

$\left(P S_{9}\right)$ practically quasistable if for given $(\lambda, B, T)$ with $0<\lambda<A, B>0, T>0$, and some $t_{0} \in R_{+}$, we have that $u_{0}<\lambda$ implies $u(t)<B$, for $t \geq t_{0}+T$

$\left(P S_{10}\right)$ uniformly practically quasistable if $\left(P S_{9}\right)$ holds for all $t_{0} \in R_{+}$ 
Theorem 1. Assume that the following conditions hold $\left(A_{3}\right)\left(q(t, x), T_{k}, \lambda, A\right)$ with $0<\lambda<A$ are given $\left(A_{4}\right)$ there exists a function $V \in C\left(T_{k} \times D(A), R_{+}\right)$and $V \in \Lambda$ such that

(i) for any $t>t_{0}, V(t, x(t))>V(t+s, x(t+s)), s \in[-\tau$, $0)$, the inequality

$$
D_{(2.1)}^{+} V(t, x(t)) \leq g(t, V(t, x(t))),
$$

holds, where $g \in C\left(T_{k} \times R_{+}, R\right)$ and $g(t, 0) \equiv 0$

(ii) $b(\|q(t, x)\|) \leq V(t, x) \leq a(\|x\|)$, where $a(\cdot), b(\cdot) \in K$ and $a(\lambda)<b(A)$

Then, equation (7) is (uniformly) practically stable with respect to $(a(\lambda), b(A))$ implies that system (1) is (uniformly) practically stable with respect to $\left(q(t, x), T_{k}, \lambda, A\right)$.

Proof. Assume that $u\left(t ; t_{0}, u_{0}\right)$ is a solution of the equation (7), and is practically stable with respect to $(a(\lambda), b(A))$ for given $0<\lambda<A$. Let $m(t)=V(t, x(t))$, where $x(t)$ is a solution of the systems (1). From the condition (i) of $\left(A_{4}\right)$, it follows that $D^{+} m(t) \leq g(t, m(t))$, for $t \geq t_{0}$. Let

$$
u\left(t_{0}\right)=\max _{s \in[-\tau, 0]} V\left(t_{0}+s, \varphi(s)\right)=\max _{s \in[-\tau, 0]} m\left(t_{0}+s\right) .
$$

By Lemma 1, we know that the inequality $V(t, x(t))=$ $m(t) \leq r(t)$, for $t \geq t_{0}$, holds, where $r(t)$ is the maximal solution of comparison equation (7) existing on $T_{k}$. Assume that $\max _{s \in[-\tau, 0]}\|\varphi(s)\|<\lambda$, then, we have

$$
\begin{aligned}
u\left(t_{0}\right) & =\max _{s \in[-\tau, 0]} V\left(t_{0}+s, \varphi(s)\right) \leq \max _{s \in[-\tau, 0]} a(\|\varphi(s)\|) \\
& =a\left(\max _{s \in[-\tau, 0]}\|\varphi(s)\|\right)<a(\lambda) .
\end{aligned}
$$

Furthermore, from the condition (ii) of $\left(A_{4}\right)$ and Lemma 1 , we get $b(\|q(t, x)\|) \leq V(t, x)=m(t) \leq r(t)<b(A)$. Thus, $\max _{s \in[-\tau, 0]}\|\varphi(s)\|<\lambda$ implies $\|q(t, x)\|<A, t \geq t_{0}$, that is, system (1) is practically stable with respect to $(q(t, x)$, $\left.T_{k}, \lambda, A\right)$.

Similarly, we can prove that equation (7) is uniformly practically stable with respect to $(a(\lambda), b(A))$ implies that the systems $(1)$ is uniformly practically stable with respect to $\left(q(t, x), T_{k}, \lambda, A\right)$. The proof is completed.

By Theorem 1, we can obtain the following corollaries.

Corollary 1. Assume that the conditions $\left(A_{3}\right)$ and (ii) of $\left(A_{4}\right)$ hold in Theorem 1, and

$\left(A_{5}\right)$ there exists a function $V \in C\left(T_{k} \times D(A), R_{+}\right)$and $V \in \Lambda$ such that for any $t>t_{0}, V(t, x(t))>V(t+s, x$ $(t+s)), s \in[-\tau, 0)$, the inequality

$$
D_{(2.1)}^{+} V \leq 0
$$

holds. Then, system (1) is uniformly practically stable with respect to $\left(q(t, x), T_{k}, \lambda, A\right)$.

The conclusion of Corollary 1 can be obtained by considering the case of $g(t, u) \equiv 0$ and $\dot{u}=0$ is uniformly practically stable with respect to $(a(\lambda), b(A))$ for given $0<\lambda<A$.

Corollary 2. Assume that the conditions $\left(A_{3}\right)$ and (ii) of $\left(A_{4}\right)$ hold in Theorem 1, and

$\left(A_{6}\right)$ there exists a function $V(t, x) \in C\left(T_{k} \times D(A), R_{+}\right)$ and $V \in \Lambda$ such that for any $t>t_{0}, V(t, x(t))>V(t+$ $s, x(t+s)), s \in[-\tau, 0)$, the inequality

$$
D_{(2.1)}^{+} V \leq \alpha(t) F(V(t, x))
$$

holds, where $F \in C\left(R_{+}, R_{+}\right)$and $0<F(V) \leq V$

$\left(A_{7}\right)$ the inequalities

$$
\int_{t_{0}}^{t} \alpha(t) \mathrm{d} t \leq M<+\infty, \quad M \leq \ln \frac{b(A)}{a(\lambda)}, t \in\left[t_{0},+\infty\right),
$$

hold

Then, system (1) is uniformly practically stable with respect to $\left(q(t, x), T_{k}, \lambda, A\right)$.

Proof. By Theorem 1, we only prove that the system $\dot{u}=$ $(\alpha(t) F(u))$ is uniformly practically stable with respect to $(a(\lambda), b(A))$. In fact, let $u\left(t_{0}\right)=u\left(t_{0} ; t_{0}, u_{0}\right)=\max _{s \in[-\tau, 0]}$ $V\left(t_{0}+s, \varphi(s)\right)$. Assume that $\max _{s \in[-\tau, 0]}\|\varphi(s)\|<\lambda$, it follows from the condition (ii) of $\left(A_{4}\right)$ that

$$
u\left(t_{0} ; t_{0}, u_{0}\right) \leq \max _{s \in[-\tau, 0]} a(\|\varphi(s)\|)=a\left(\max _{s \in[-\tau, 0]}\|\varphi(s)\|\right)<a(\lambda) .
$$

Furthermore, by condition $\left(A_{7}\right)$, the inequality

$$
u\left(t ; t_{0}, u_{0}\right) \leq u_{0} \exp \left\{\int_{t_{0}}^{t} \alpha(s) \mathrm{d} s\right\}<a(\lambda) e^{M}<b(A),
$$

holds. Then, system (1) is uniformly practically stable with respect to $\left(q(t, x), T_{k}, \lambda, A\right)$. The proof is completed.

Corollary 3. Assume that the conditions $\left(A_{3}\right)$ and (ii) of $\left(A_{4}\right)$ hold in Theorem 1, and

$\left(A_{8}\right)$ there exists a function $V \in C\left(T_{k} \times D(A), R_{+}\right)$and $V \in \Lambda$ such that for any $t>t_{0}, V(t, x(t))>V(t+s, x$ $(t+s))$ for $s \in[-\tau, 0)$, the inequality 


$$
D_{(2.1)}^{+} V \leq-\alpha F(V(t, x))+\beta
$$

holds, in which $\alpha$ and $\beta$ are positive constants, $0<F(V) \leq V$

$\left(A_{9}\right)$ the inequalities

$$
\begin{aligned}
a(\lambda) & <b(A), \\
a(\lambda)+\frac{\beta}{\alpha} & \leq b(A),
\end{aligned}
$$

hold

Then, system (1) is uniformly practically stable with respect to $\left(q(t, x), T_{k}, \lambda, A\right)$.

Proof. In fact, we only need to prove that the system $\dot{u}=$ $-\alpha F(u)+\beta$ is uniformly practically stable with respect to $(a(\lambda), b(A))$. Let $u\left(t_{0}\right)=u\left(t_{0} ; t_{0}, u_{0}\right)=\max _{s \in[-\tau, 0]} V\left(t_{0}+\right.$ $s, \varphi(s))$; then, we have

$$
u\left(t_{0} ; t_{0}, u_{0}\right) \leq \max _{s \in[-\tau, 0]} a(\|\varphi(s)\|)=a\left(\max _{s \in[-\tau, 0]}\|\varphi(s)\|\right)<a(\lambda) .
$$

Furthermore, by condition $\left(A_{8}\right)$, the inequality

$$
u\left(t ; t_{0}, u_{0}\right) \leq u_{0} e^{-\alpha\left(t-t_{0}\right)}+\frac{\beta}{\alpha}<a(\lambda)+\frac{\beta}{\alpha} \leq b(A)
$$

holds. Then, system (1) is uniformly practically stable with respect to $\left(q(t, x), T_{k}, \lambda, A\right)$.

Theorem 2. Assume that the conditions $\left(A_{3}\right)$ and (ii) of $\left(A_{4}\right)$ hold in Theorem 1, and (iii) $V(t, x) \geq b(\|q(t, x)\|)$, $V\left(t_{0}, x\right) \leq a\left(t_{0},\|x\|\right)$, where $b(\cdot) \in K, a\left(t_{0}, \cdot\right) \in K^{*}$.

Then, equation (7) is (uniformly) practically stable with respect to $\left(a\left(t_{0}, \lambda\right), b(A)\right)$ implies that the systems $(1)$ is (uniformly) practically stable with respect to $(q(t, x)$, $\left.T_{k}, \lambda, A\right)$.

Proof. In fact, by the condition (iii) of $\left(A_{4}\right)$, we have

$$
\begin{aligned}
u\left(t_{0}\right) & =\max _{s \in[-\tau, 0]} V\left(t_{0}+s, \varphi(s)\right) \leq \max _{s \in[-\tau, 0]} a\left(t_{0},\|\varphi(s)\|\right) \\
& =a\left(t_{0}, \max _{s \in[-\tau, 0]}\|\varphi(s)\|\right) .
\end{aligned}
$$

Then, we can get the result by using a method similar to Theorem 1. We omit its details.

Theorem 3. Assume that the following conditions hold

$\left(A_{10}\right)\left(q(t, x), T_{k}, \lambda, A, B, T\right)$ with $0<\lambda<A, 0<B<A$ and $T>0$ are given

$\left(A_{4}\right)$ there exists a function $V \in C\left(T_{k} \times D(A), R_{+}\right)$and $V \in \Lambda$ such that

(i) for any $t>t_{0}, V(t, x(t))>V(t+s, x(t+s)), s \in[-\tau$, $0)$, the inequality

$$
D_{(2.1)}^{+} V(t, x(t)) \leq g(t, V(t, x(t)))
$$

holds, where $g \in C\left(T_{k} \times R_{+}, R\right), g(t, 0) \equiv 0$

(ii) $b(\|q(t, x)\|) \leq V(t, x) \leq a(\|x\|)$, where $a(\cdot), b(\cdot) \in K$ and $a(\lambda)<b(A)$

Then, equation (7) is (uniformly) practically quasistable with respect to $(a(\lambda), b(B), T)$ implies that system (1) is (uniformly) practically quasistable with respect to $\left(q(t, x), T_{k}, \lambda, B, T\right)$.

Proof. Assume that $u\left(t ; t_{0}, u_{0}\right)$ is a solution of equation (7) and is practically quasistable with respect to $(a(\lambda), b(B), T)$ for given $0<\lambda<A, \quad 0<B<A$ and $T>0$. Let $m(t)=$ $V(t, x(t))$, where $x(t)$ is a solution of system (1). It follows from the condition $\left(A_{4}\right)$ that

$$
D^{+} m(t) \leq g(t, m(t)), \quad \text { for } t \geq t_{0} .
$$

Let $\quad u\left(t_{0}\right)=\max _{s \in[-\tau, 0]} V\left(t_{0}+s, \varphi(s)\right)=\max _{s \in[-\tau, 0]}$ $m\left(t_{0}+s\right)$. Then, by Lemma 1 , we know that the inequality

$$
V(t, x)=m(t) \leq r(t), \quad \text { for } t \geq t_{0},
$$

holds, where $r(t)$ is the maximal solution of comparison equation (7) existing on $T_{k}$. Assume that $\varphi(s) \in C([-\tau, 0]$, $R^{n}$ ) and $\max _{s \in[-\tau, 0]}\|\varphi(s)\|<\lambda$. Then, we obtain

$$
\begin{aligned}
u\left(t_{0}\right) & =\max _{s \in[-\tau, 0]} V\left(t_{0}+s, \varphi(s)\right) \leq \max _{s \in[-\tau, 0]} a(\|\varphi(s)\|) \\
& =a\left(\max _{s \in[-\tau, 0]}\|\varphi(s)\|\right)<a(\lambda)
\end{aligned}
$$

Furthermore, by comparison equation (7) which is practically quasistable with respect to $(a(\lambda), b(B), T)$, the condition $\left(A_{4}\right)$, and Lemma 1 , we get

$b(\|q(t, x)\|) \leq V(t, x)=m(t) \leq r(t)<b(B), \quad$ for $t \geq t_{0}+T$.

Thus, $\max _{s \in[-\tau, 0]}\|\varphi(s)\|<\lambda$ implies $\|q(t, x)\|<B$, $t \geq t_{0}+T$, that is, system (1) is practically quasistable with respect to $\left(q(t, x), T_{k}, \lambda, B, T\right)$.

Similarly, we can prove that equation (7) is uniformly practically stable with respect to $(a(\lambda), b(B), T)$ implies that the systems (1) is uniformly practically stable with respect to $\left(q(t, x), T_{k}, \lambda, B, T\right)$.

Theorem 4. Assume that the conditions $\left(A_{10}\right)$ and $(i)$ of $\left(A_{4}\right)$ hold in Theorem 3, and the condition (ii) of $\left(A_{4}\right)$ is replaced by

(iv) $V(t, x) \geq b(\|q(t, x)\|), V\left(t_{0}, x\right) \leq a\left(t_{0},\|x\|\right)$, where $b(\cdot) \in K, a\left(t_{0}, \cdot\right) \in K^{*}$

Then, equation (7) is (uniformly) practically quasistable with respect to $(a(\lambda), b(B), T)$ implies that the systems (1) is (uniformly) practically quasistable with respect to $\left(q(t, x), T_{k}, \lambda, B, T\right)$.

The proof of Theorem 4 is similar to that of Theorem 3, so we omit its details. 


\section{Integral Stability}

The concept of integral stability, which was introduced for ordinary differential equations by Vrhoc in 1959 [26] and Lakshmikantham in 1969 [27], enlarges the collection of dynamical properties of solutions which can be investigated by the direct Lyapunov method. The integral stability theory has been rapidly developed recently. For example, Martynyuk [28], Salvadori and Visentin [29], Soliman and Abdalla [30] obtained the integral stability criteria for nonlinear differential equations, respectively; Hristova [31] obtained the integral stability in terms of two measures for impulsive differential equations; and Sood and Srivastava [32] gave the $\varphi_{0}$-integral stability criteria for impulsive differential equations. The main purpose of this section is to discuss the integral stability of singular differential systems with maxima and its perturbed systems.

Consider singular differential system (1) and its perturbed systems

$$
\begin{cases}E \dot{x}=f\left(t, x, \max _{s \in[t-\tau, t]} x(s)\right)+h\left(t, x, \max _{s \in[t-\tau, t]} x(s)\right), & \text { for } t \geq t_{0} \geq 0, \\ x_{t_{0}}=\varphi(t), & t \in[-\tau, 0],\end{cases}
$$

where $h \in C\left(R_{+} \times R^{n} \times R^{n}, R^{n}\right), h(t, 0,0) \equiv 0$.

Let $S_{p k}\left(t_{0}\right)$ be a set of all consistent initial functions of (1) and (26) in $\left[t_{0}, t_{k}\right)$ through $\left(t_{0}, \varphi\right)$. For any $\varphi \in S_{p k}\left(t_{0}\right)$, assume that there exists a continuous solution of (1) and (26) in $\left[t_{0}, t_{k}\right)$ through $\left(t_{0}, \varphi\right)$ at least.

$$
\begin{aligned}
& B(\varphi, \delta)=\left\{\psi \in C\left([-\tau, 0], R^{n}\right) \mid \max _{s \in[-\tau, 0]}\|\psi(s)-\varphi(s)\|\right. \\
& \left.<\delta, \varphi \in C\left([-\tau, 0], R^{n}\right), \delta \in R_{+}\right\} .
\end{aligned}
$$

Definition 5. Let $\varphi \in S_{p k}\left(t_{0}\right)$. Singular system (1) is said to be $\left(I S_{1}\right)$ equi-integrally stable on $\left\{q(t, x), T_{k}\right\}$, if for given $\alpha \geq 0$ and $t_{0} \in T_{k}$, there exists a positive function $\beta=\beta\left(t_{0}, \alpha\right)$, which is continuous in $t_{0}$ for each $\alpha$ and $\beta \in K$, such that, for every solution $x\left(t ; t_{0}, \varphi\right)$ of the perturbed systems (26),

$$
q(t, x) \in D(\beta), \quad t \geq t_{0},
$$

holds, provided that $\varphi \in B(0, \alpha) \cap S_{p k}\left(t_{0}\right)$ and

$$
\int_{t_{0}}^{t_{0}+T} \sup _{\|q(t, x)\| \leq \beta}\left\|h\left(s, x, \max _{u \in[s-\tau, s]} x(u)\right)\right\| \mathrm{d} s \leq \alpha,
$$

for $T>0$;

$\left(I S_{2}\right)$ uniformly integrally stable on $\left\{q(t, x), T_{k}\right\}$, if the $\beta$ in $\left(I S_{1}\right)$ is independent of $t_{0}$

$\left(I S_{3}\right)$ equiasymptotically integrally stable on $\left\{q(t, x), T_{k}\right\}$, if $\left(I S_{1}\right)$ holds and for every $\epsilon>0, \alpha \geq 0$ and $t_{0} \in T_{k}\left(t_{k}=+\infty\right)$, there exist positive functions $T=$
$T\left(t_{0}, \alpha, \epsilon\right)$ and $\gamma=\gamma\left(t_{0}, \alpha, \epsilon\right)$, which are continuous in $t_{0}$ for each $\alpha$ and $\epsilon$, and for every solution $x\left(t ; t_{0}, \varphi\right)$ of the perturbed systems (26),

$$
q(t, x) \in D(\epsilon), \quad t \geq t_{0}+T
$$

holds, provided that $\varphi \in B(0, \alpha) \cap S_{p}\left(t_{0}\right)$, and

$$
\int_{t_{0}}^{+\infty} \sup _{\|q(t, x)\| \leq \beta}\left\|h\left(s, x, \max _{u \in[s-\tau, s]} x(u)\right)\right\| \mathrm{d} s \leq \gamma,
$$

$\left(I S_{4}\right)$ uniformly asymptotically integrally stable on $\left\{q(t, x), T_{k}\right\}$ if the $T$ and $\gamma$ in $\left(I S_{3}\right)$ are independent of $t_{0}$ and $\left(I S_{2}\right)$ holds

Now, we consider comparison scalar differential equation (7) and its perturbed equation

$$
\dot{u}=g(t, u)+p(t), \quad u\left(t_{0}\right)=u_{0},
$$

where $g(t, 0) \equiv 0, g \in C\left[T_{k} \times R_{+}, R\right], p(t) \in C\left[T_{k}, R_{+}\right]$.

Definition 6. Equation (7) is said to be equi-integrally stable, if for given $\alpha_{1} \geq 0, t_{0} \in T_{k}$, there exists a positive function $\beta_{1}=\beta_{1}\left(t_{0}, \alpha_{1}\right)$ that is continuous in $t_{0}$ for each $\alpha_{1}$ and $\beta_{1} \in K$, such that, for every solution $u\left(t ; t_{0}, u_{0}\right)$ of the perturbed differential equation (32), the inequality

$$
\left|u\left(t ; t_{0}, u_{0}\right)\right|<\beta_{1},
$$

holds, provided that $\left|u_{0}\right| \leq \alpha_{1}$ and $\int_{t_{0}}^{t_{0}+T} p(s) \mathrm{d} s \leq \alpha_{1}$ for every $T>0$.

Remark 2. Similar to Definition 5, we can give the corresponding concepts of stability of equation (7).

Next, we investigate the integral stability of system (1) via the Lyapunov function method and comparison principle.

Theorem 5. Assume that the condition (i) of $\left(A_{4}\right)$ holds in Theorem 1, and condition (ii) of $\left(A_{4}\right)$ is replaced by

$$
\begin{aligned}
\left(A_{4}^{\prime}\right) b(\|q(t, x)\|) & \leq V(t, x), \quad t \geq t_{0}, \text { where } b \in K, \\
& \text { and } b(r) \longrightarrow+\infty \text { as } r \longrightarrow+\infty .
\end{aligned}
$$

Then, equation (7) which is equi-integrally stable implies that system (1) is equi-integrally stable on $\left\{q(t, x), T_{k}\right\}$.

Proof. Let $\varphi \in B(0, \alpha) \cap S_{p k}\left(t_{0}\right)$ for every $\alpha \geq 0, t_{0} \in T_{k}$. Since $V(t, x)$ is Lipschitzian in $x$, we have

$$
\begin{gathered}
|V(t, x)-V(t, y)| \leq L\|x-y\|, \quad L>0 \text { is a constant, } \\
\max _{s \in[-\tau, 0]} V\left(t_{0}+s, \varphi(s)\right) \leq L \max _{s \in[-\tau, 0]}\|\varphi(s)\| \leq L \alpha=\alpha_{1} .
\end{gathered}
$$

Let $x(t)=x\left(t ; t_{0}, \varphi\right)$ be any solution of (26). Thus, by the condition (i) of $\left(A_{4}\right)$ and (29), we get

$$
D_{(21)}^{+} V(t, x) \leq g(t, V(t, x))+L\left\|h\left(t, x, \max _{s \in[t-\tau, t]} x(s)\right)\right\| .
$$


Defining $p(t)=L\left\|h\left(t, x, \max _{s \in[t-\tau, t]} x(s)\right)\right\|$ and choosing $u_{0}=\max _{s \in[-\tau, 0]} V\left(t_{0}+s, \varphi(s)\right)$, by Lemma 1 , we have

$$
V(t, x) \leq r\left(t ; t_{0}, u_{0}\right)
$$

where $r\left(t ; t_{0}, u_{0}\right)$ is the maximal solution of (32).

If equation (7) is equi-integrally stable, then for $\alpha_{1} \geq 0$ and $t_{0} \in T_{k}$, there exists a $\beta_{1}=\beta_{1}\left(t_{0}, \alpha_{1}\right)$, which is continuous in $t_{0}$ for each $\alpha_{1}$ and $\beta_{1} \in K$, such that, for every solution $u\left(t ; t_{0}, u_{0}\right)$ of (32), the inequality

$$
u\left(t ; t_{0}, u_{0}\right)<\beta_{1}, \quad t \geq t_{0}
$$

holds, whenever $u_{0} \leq \alpha_{1}$ and $\int_{t_{0}}^{t_{0}+T} p(s) \mathrm{d} s \leq \alpha_{1}$ for any $T>0$.

By the condition $\left(A_{4}^{\prime}\right)$, it is possible to choose a $\beta=$ $\beta\left(t_{0}, \alpha\right)$ satisfying

$$
b(\beta) \geq \beta_{1} .
$$

It is easily shown that $\beta$ is continuous in $t_{0}$ for each $\alpha$ and $\beta \in K$ for each $t_{0} \in T_{k}$. Moreover, we claim that system (1) is equi-integrally stable on $\left\{q(t, x), T_{k}\right\}$. In fact, if this is not true, there exists a $t_{1}>t_{0}$ such that

$$
\left\|q\left(t_{1}, x\right)\right\|=\beta \text {, and }\|q(t, x)\|<\beta, t \in\left[t_{0}, t_{1}\right) .
$$

From (37)-(40), we have

$$
\beta_{1} \leq b(\beta) \leq V\left(t_{1}, x\left(t_{1} ; t_{0}, \varphi\right)\right) \leq r\left(t_{1} ; t_{0}, u_{0}\right)<\beta_{1},
$$

which is a contradiction. Thus, system (1) is equi-integrally stable on $\left\{q(t, x), T_{k}\right\}$.

Corollary 4. Assume that the conditions of Theorem 5 hold. Then, equation (7) which is uniformly integrally stable implies that system (1) is uniformly integrally stable on $\left\{q(t ; x) ; T_{k}\right\}$.

The detailed proof of Corollary 4 is similar to the proof in Theorem 5, so we omit it.

Theorem 6. Assume that the conditions of Theorem 5 hold and $t_{k}=+\infty$ Then, equation (7) which is equiasymptotically integrally stable implies that system (1) is equiasymptotically integrally stable on $\left\{q(t . x), R_{+}\right\}$.

Proof. It can be known from the proof of Theorem 5 that system (1) is equi-integrally stable on $\left\{q(t, x), R_{+}\right\}$. Let $\varphi \in B(0, \alpha) \cap S_{p}\left(t_{0},+\infty\right)$ and $\alpha_{1}=L \alpha$, for given $\alpha \geq 0$ and $t_{0} \in[0,+\infty)$. For given .., $\alpha_{1} \geq 0$ and $t_{0} \in R_{+}$, there exist $\gamma_{1}=$ $\gamma_{1}\left(t_{0}, \alpha_{1}, \varepsilon\right)$ and $T=T\left(t_{0}, \alpha_{1}, \varepsilon\right)$, such that for every solution $u\left(t ; t_{0}, u_{0}\right)$ of $(32)$,

$$
u\left(t ; t_{0}, u_{0}\right)<b(\varepsilon), t \geq t_{0}+T,
$$

holds, whenever $u_{0}=\max _{s \in[-\tau, 0]} V\left(t_{0}+s, \varphi(s)\right) \leq \alpha_{1}$ and $\int_{t_{0}}^{\infty} p(s) \mathrm{d} s<\gamma_{1}$.

Choosing a positive number $\gamma=\gamma\left(t_{0}, \alpha, \epsilon\right)$ such that $L \gamma=\gamma_{1}$, for given $\gamma$ and $T$, system (1) satisfies $\left(I S_{3}\right)$ of Definition 5. In fact, suppose that the conclusion is not true, then $q(t, x) \in D(\epsilon), t \geq t_{0}+T$ cannot be satisfied when

$$
\begin{aligned}
& \varphi \in B(0, \alpha) \cap S_{p}\left(t_{0},+\infty\right) \\
& \text { and } \int_{t_{0}}^{+\infty} \sup _{\|q(t, x)\| \leq \beta}\left\|h\left(s, x, \max _{u \in[s-\tau, s]} x(u)\right)\right\| \mathrm{d} s \leq \gamma .
\end{aligned}
$$

Let $\left\{t_{k}\right\}$ be a sequence such that $t_{k} \geq t_{0}+T$ and $\lim _{k \longrightarrow+\infty t k}=+\infty$. Suppose that there is a solution $x(t)=$ $x\left(t ; t_{0}, \varphi\right)$ of system (26), such that for every $k$,

$$
\left\|q\left(t_{k}, x\left(t_{k} ; t_{0}, \varphi\right)\right)\right\| \geq \varepsilon .
$$

By the condition $\left(A_{4}^{\prime}\right)$ and (44), we obtain

$$
b(\varepsilon) \leq b\left(\left\|q\left(t_{k}, x\left(t_{k} ; t_{0}, \varphi\right)\right)\right\|\right) \leq V\left(t_{k}, x\left(t_{k} ; t_{0}, \varphi\right)\right) \text {. }
$$

Furthermore, by the equiasymptotical integral stability of equations (7), (37), and (42)-(59), we can get

$$
b(\epsilon) \leq V\left(t_{k}, x\left(t_{k} ; t_{0}, \varphi\right)\right) \leq r\left(t_{k} ; t_{0}, u_{0}\right)<b(\epsilon) .
$$

which is a contradiction. Thus, system (1) is equiasymptotically integrally stable on $\{q(t, x),[0,+\infty)\}$.

Corollary 5. Assume that the conditions of Theorem 6 hold. Then, equation (7) which is uniformly asymptotically integrally stable implies that system (1) is uniformly asymptotically integrally stable on $\left\{q(t ; x) ; T_{k}\right\}$.

In fact, we can show that the positive numbers $T$ and $\gamma_{1}$ in proof of Theorem 6 are independent of $t_{0}$; therefore, (44) implies that $\gamma$ is independent of $t_{0}$. The rest of the proof is similar to that of Theorem 6 , so we omit the details here.

For the comparison equation (7), if we suppose that the function $g(t, u)$ is nonincreasing in $u$ for $t \in\left[t_{0},+\infty\right)$; then, we can get the uniform asymptotic integral stability of system (1) by the uniform asymptotic stability of the comparison equation (7). Therefore, we firstly give the following definition and Lemmas, which can be found in [27].

Definition 7. Comparison equation (7) is said to be

$\left(S_{1}\right)$ equistable, if for each $\epsilon>0, t_{0} \in R_{+}$, there exists a positive function $\delta=\delta\left(t_{0}, \epsilon\right)$, such that

$$
\left|u_{0}\right| \leq \delta \Longrightarrow\left|u\left(t ; t_{0}, u_{0}\right)\right|<\varepsilon, \quad \text { for } t \geq t_{0},
$$

$\left(S_{2}\right)$ uniformly stable if $\delta$ in $\left(S_{1}\right)$ is independent of $t_{0}$ $\left(S_{3}\right)$ equiasymptotically stable, if it is equistable and for each $\epsilon>0, t_{0} \in R_{+}$, there exists a positive function $\delta_{0}=\delta_{0}\left(t_{0}\right), T=T\left(t_{0}, \epsilon\right)$, such that

$$
\left|u_{0}\right| \leq \delta_{0} \Longrightarrow\left|u\left(t ; t_{0}, u_{0}\right)\right|<\epsilon, \text { for } t \geq t_{0}+T,
$$

$\left(S_{4}\right)$ uniformly asymptotically stable if $\left(S_{2}\right)$ holds and the numbers $\delta_{0}$ and $T$ in $\left(S_{3}\right)$ are independent of $t_{0}$

Lemma 2 (see [27]). Equation (7) is uniformly stable if and only if there exists a function $a(r) \in K$, such that

$$
\left|u_{0}\right| \leq \delta \Longrightarrow\left|u\left(t ; t_{0}, u_{0}\right)\right| \leq a\left(\left|u_{0}\right|\right), \quad \text { for } t \geq t_{0} .
$$


Lemma 3 (see [27]). Equation (7) is uniformly asymptotically stable if and only if there exist functions $a(r) \in K$ and $\sigma(r) \in \mathcal{S}$, such that

$$
\left|u_{0}\right| \leq \delta \Longrightarrow\left|u\left(t ; t_{0}, u_{0}\right)\right| \leq a\left(\left|u_{0}\right|\right) \sigma\left(t-t_{0}\right), \quad \text { for } t \geq t_{0},
$$

where $\mathscr{F}=\left\{\sigma(r) \in C\left[R_{+}, R_{+}\right] \sigma(r)\right.$ is monotone decreasing in $r$, and $\sigma(r) \longrightarrow 0$ as $r \longrightarrow+\infty\}$.

Theorem 7. Assume that the conditions of Theorem 6 hold, the function $g(t ; u)$ be nonincreasing in $u$ for any $t \in\left[t_{0},+\infty\right)$, and

$$
\begin{array}{r}
V(t, x(t))>V(t+s, x(t+s))-L \int_{t}^{t+s}\left\|h\left(v, x, \max _{u \in[v-\tau, v]} x(u)\right)\right\| \mathrm{d} v, \\
\text { for } s \in[-\tau, 0) .
\end{array}
$$

Then, equation (7) which is uniformly asymptotically stable implies that system (1) is uniformly asymptotically integrally stable on $\left\{q(t, x), T_{k}\right\}$.

Proof. Firstly, we prove system (1) is uniformly integrally stable. Because equation (7) is uniformly stable, by Lemma 2 , there exists a function $\beta_{1} \in K$, such that

$$
0<u_{0} \leq \alpha_{1} \text { implies } u\left(t ; t_{0}, u_{0}\right)<\beta_{1}\left(u_{0}\right), \quad t \geq t_{0} .
$$

For given $\alpha>0$ and $t_{0} \in T_{k}$, where $L \alpha=\alpha_{1}$, let $\varphi \in B(0, \alpha) \cap S_{p}\left(t_{0}\right)$. Since $V(t, x)$ is Lipschitzian in $x$, we have

$$
\max _{s \in[-\tau, 0]} V\left(t_{0}+s, \varphi(s)\right) \leq L \max _{s \in[-\tau, 0]}\|\varphi(s)\| \leq L \alpha=\alpha_{1} .
$$

Let $x(t)=x\left(t ; t_{0}, \varphi\right)$ be any solution of (26) with $\varphi \in B(0, \alpha) \cap S_{p}\left(t_{0}\right)$, and

$$
V\left(t, x\left(t ; t_{0}, \varphi\right)\right) \equiv m(t)+p(t),
$$

where $p(t)=L \int_{t_{0}}^{t}\left\|h\left(s, x, \max _{u \in[s-\tau, s]} x(u)\right)\right\| \mathrm{d} s$. According to the condition (i) of $\left(A_{4}\right)$, we get

$$
\begin{aligned}
D^{+} m(t) & \leq D_{(3.1)}^{+} V(t, x(t))-L\left\|h\left(t, x, \max _{s \in[t-\tau, t]} x(s)\right)\right\| \\
& \leq D_{(2.1)}^{+} V(t, x(t)) \leq g(t, m(t)) .
\end{aligned}
$$

By (51) and Lemma 1, we have

$$
m(t) \leq r\left(t ; t_{0}, u_{0}\right),
$$

where $r\left(t ; t_{0}, u_{0}\right)$ is the maximal solution of (7) with $u_{0}=\max _{s \in[-\tau, 0]} m\left(t_{0}+s\right)$.

We choosing $\beta>0$, such that

$$
b(\beta)>\beta_{1}(L \alpha)+L \alpha .
$$

In view of the condition $b(r) \longrightarrow+\infty$ as $r \longrightarrow+\infty$, the choice of $\beta$ is reasonable. It is obvious that $\beta=\beta(\alpha)$ and $\beta \in K$. At the same time, we can claim that system (1) is uniformly integrally stable. In other words, the solution of system (26) satisfies $q(t, x) \in D(\beta), \quad t \geq t_{0}$, whenever $\varphi \in B(0, \alpha) \cap S_{p}\left(t_{0}\right)$, and

$$
\int_{t_{0}}^{t_{0}+T} \sup _{\|q(t, x)\| \leq \beta}\left\|h\left(s, x, \max _{u \in[s-\tau, s]} x(u)\right)\right\| \mathrm{d} s \leq \alpha, \quad \text { for } T>0 .
$$
that

Suppose that this is not true; there exists a $t_{1}>t_{0}$ such

$\left\|q\left(t_{1}, x\left(t_{1}\right)\right)\right\|=\beta$ and $\|q(t, x(t))\| \leq \beta, t \in\left[t_{0}, t_{1}\right]$.

From the condition $\left(A_{4}^{\prime}\right)$ and (54)-(58), we get

$$
\begin{aligned}
b(\beta) & \leq V\left(t_{1}, x\left(t_{1}\right)\right) \leq r\left(t_{1} ; t_{0}, u_{0}\right)+p\left(t_{1}\right) \\
& \leq \beta_{1}(L \alpha)+L \int_{t_{0}}^{t_{1}} \sup _{\|q(t, x)\| \leq \beta}\left\|h\left(s, x, \max _{u \in[s-\tau, s]} x(u)\right)\right\| \mathrm{d} s \\
& \leq \beta_{1}(L \alpha)+L \alpha<b(\beta) .
\end{aligned}
$$

This is a contradiction, and then system (1) is uniformly integrally stable.

Secondly, we prove that system (1) is uniformly asymptotically integrally stable. By the uniform asymptotic stability of equation (7) and Lemma 3, we have

$$
u\left(t ; t_{0}, u_{0}\right) \leq \beta_{1}\left(u_{0}\right) \sigma\left(t-t_{0}\right), \quad t \geq t_{0},
$$

where $\beta_{1} \in K$ and $\sigma \in \mathscr{F}$. For given $\epsilon>0, \alpha \geq 0$ and $t_{0} \in T_{k}$, let $\varphi \in B(0, \alpha) \cap S_{p}\left(t_{0}\right)$, and

$$
L \int_{t_{0}}^{+\infty} \sup _{\|q(t, x)\| \leq \beta}\left\|h\left(s, x, \max _{u \in[s-\tau, s]} x(u)\right)\right\| \mathrm{d} s \leq L \gamma_{1}<b(\varepsilon),
$$

where $\gamma=\min \left(\gamma_{1}, \alpha\right)$. For any solution $x(t)=x\left(t ; t_{0}, \varphi\right)$ of (26) and (56), holds whenever $u_{0}=\max V\left(t_{0}+s, \varphi(s)\right)$. By (54), (56), and (61), together with ${ }^{s \notin}\left[A_{4}^{t}\right)$, , we can obtain the inequality

$$
\begin{aligned}
b(\|q(t, x(t))\|) \leq & V(t, x(t)) \leq r\left(t ; t_{0}, u_{0}\right) \\
& +L \int_{t_{0}}^{t}\left\|h\left(s, x, \max _{u \in[s-\tau, s]} x(u)\right) \mathrm{d} s\right\| \\
\leq & \beta_{1}(L \alpha) \sigma\left(t-t_{0}\right) \\
& +L \int_{t_{0}}^{t} \sup _{\|(t, x)\| \leq \beta}\left\|h\left(s, x \max _{u \in[s-\tau, s]} x(u)\right)\right\| \mathrm{d} s \\
< & \beta_{1}(L \alpha) \sigma\left(t-t_{0}\right)+L \gamma .
\end{aligned}
$$

Since $\sigma \in \mathscr{F}$, then there exists a $T=T(\alpha, \epsilon)$, such that

$$
\sigma\left(t-t_{0}\right)<\frac{b(\epsilon)-L \gamma}{\beta_{1}(L \alpha)}, \quad t \geq t_{0}+T .
$$

Furthermore, we have

$$
b(\|q(t, x(t))\|)<b(\epsilon), \quad t \geq t_{0}+T,
$$

which implies that 


$$
\|q(t, x(t))\|<\epsilon, \quad t \geq t_{0}+T
$$

provided $\varphi \in B(0, \alpha) \cap S_{p}\left(t_{0}\right)$, and (62) is satisfied. Therefore, system (1) is uniformly asymptotically integrally stable.

\section{Conclusion}

This paper discussed a class of nonlinear singular differential systems with maxima. Some notions of practical stability and integral stability for such systems were introduced, and various stability criteria were obtained by using the Lyapunov method and comparison principle.

\section{Data Availability}

Data sharing is not applicable to this article as no data sets were generated or analyzed during the current study.

\section{Conflicts of Interest}

The authors declare that they have no conflicts of interest.

\section{Authors' Contributions}

All authors read and approved the final manuscript.

\section{Acknowledgments}

This paper was supported by the National Natural Science Foundation of China (11771115 and 11271106).

\section{References}

[1] D. Bainov and S. Hristova, Differential Equations with Maxima, CRC Press, New York, NY, USA, 2011.

[2] E. Popov, Automatic Regulation and Control, Elsevier, Amsterdam, Netherlands, in Russian, 1996.

[3] N. Bantsur and E. Trofimchuk, "On the existence and stability of the periodic and almost periodic solutions of quasilinear systems with maxima," Ukrainian Mathematical Journal, vol. 6, pp. 747-754, 1998, in Ukrainian.

[4] M. Bohner, A. Atanaska, and S. G. Hristova, "Nonlinear differential equations with "maxima": parametric stability in terms of two measures," Applied Mathematics \& Information Sciences, vol. 7, no. 1, pp. 41-48, 2013.

[5] T. Donchev, N. Kitanov, and D. Kolev, "Stability for the solutions of parabolic equations with maxima," PanAmerican Mathematical Journal, vol. 20, no. 2, pp. 1-19, 2010.

[6] S. Hristova, "Stability in terms of two measures for impulsive differential equations with supremum," Nonlinear Studies, vol. 17, no. 4, pp. 299-308, 2010.

[7] S. Hristova and S. Gluhcheva, "Lipschitz stability in terms of two measures for differential equations with maxima," International Journal of Pure and Applied Mathematics, vol. 2, no. 2, pp. 1-12, 2010.

[8] S. G. Hristova and A. Georgieva, "Practical stability in terms of two measures for impulsive differential equations with supremum," International Journal of Differential Equations, vol. 2011, Article ID 703189, 13 pages, 2011.

[9] P. Wang, Q. Xu, and X. Liu, "Integral stability in terms of two measures for nonlinear differential systems with maxima,"
Abstract and Applied Analysis, vol. 2014, Article ID 656290, 7 pages, 2014.

[10] S. L. Campbell, Singular Systems of Differential Equations, Pitman Advanced Publishing Program (I), London, UK, 1980.

[11] L. Dai, Singular Control Systems, Springer-Verlag, New York, NY, USA, 1989.

[12] S. L. Campbell, Singular Systems of Differential Equations, Pitman Advanced Publishing Program (II), London, UK, 1982.

[13] M. Mirko and B. Vladimir, "Stability analysis of singular systems," Circuits Systems and Signal Processing, vol. 8, no. 3, pp. 267-287, 1989.

[14] P. Anh and D. Hoang, "Stability of a class of singular difference equations," IJDE, International Journal of Difference Equations, vol. 1, pp. 181-193, 2006.

[15] K. Takaba, N. Morihira, and T. Katayama, "A generalized Lyapunov theorem for descriptor system," Systems \& Control Letters, vol. 24, no. 1, pp. 49-51, 1995.

[16] P. Wang, M. Wu, and Y. Wu, "On $\phi_{0}$-stability of a class of singular difference equations," Advances in Difference Equations, vol. 1, pp. 1-10, 2013.

[17] L. Zhang, J. Lam, and Q. Zhang, "New Lyapunov and Riccati equations for discrete time descriptor systems," IEEETransactions on Automatic Control Author Information, vol. 44, no. 11, pp. 2134-2139, 1999.

[18] Y. Li and Y. Liu, "Stability of solutions of singular systems with delay," Control Theory \& Applications, vol. 15, pp. 542-550, 1998.

[19] P. Wang and J. Zhang, "Stability of solutions for nonlinear singular systems with delay," Applied Mathematics Letters, vol. 25, no. 2, pp. 1291-1295, 2012.

[20] C. Yang, Q. Zhang, and L. Zhou, "Practical stability of descriptor systems with time delays in terms of two measurements," Journal of the Franklin Institute, vol. 343, no. 6, pp. 635-646, 2006.

[21] S. Hristova, "Practical stability and cone valued Lyapunov functions for differential equations with maxima," International Journal of Pure and Applied Mathematics, vol. 57, no. 3, pp. 313-324, 2009.

[22] V. Lakshmikantham, S. Leela, and A. Martynyuk, Practical Stability of Nonlinear Systems, World Scientific, Singapore, 1990.

[23] P. Mahajan, S. K. Srivastava, and R. Dogra, "Uniform practical stability of perturbed impulsive differential system in terms of two measures," Malaya Journal of Matematik, vol. 7, no. 2, pp. 142-146, 2019.

[24] A. Martynyuk, G. Stamov, and I. Stamova, "Practical stability analysis with respect to manifolds and boundedness of differential equations with fractional-like derivatives," Rocky Mountain Journal of Mathematics, vol. 49, no. 1, pp. 211-233, 2019.

[25] Y. Zhang and J. Sun, "Eventual practical stability of impulsive differential equations with time delay in terms of two measurements," Journal of Computational and Applied Mathematics, vol. 176, no. 1, pp. 223-229, 2005.

[26] I. Vrhoc, "Integral stability," Czechoslovak Mathematical Journal, vol. 9, pp. 71-129, 1959.

[27] V. Lakshmikantham and S. Leela, Differential and Integral Inequalities, Vol. 1, Academic Press, New York, NY, USA, 1969.

[28] A. A. Martynyuk, "On integral stability and Lipschitz stability of motion," Ukrainian Mathematical Journal, vol. 49, no. 1, pp. 84-92, 1997. 
[29] L. Salvadori and F. Visentin, "Non-asymptotic stability and integral stability trough a reduction principle," Ricerche di Matematica, vol. 63, no. 2, pp. 335-345, 2014.

[30] A. A. Soliman and M. H. Abdalla, "Integral stability criteria of nonlinear differential systems," Mathematical and Computer Modelling, vol. 48, no. 1-2, pp. 258-267, 2008.

[31] S. G. Hristova, "Integral stability in terms of two measures for impulsive functional differential equations," Mathematical and Computer Modelling, vol. 51, no. 1-2, pp. 100-108, 2010.

[32] A. Sood and S. K. Srivastava, "Integral $\Phi_{0}$-stability of impulsive differential equations," Open Journal of Applied Sciences, vol. 5, no. 10, pp. 651-660, 2015. 